\title{
Maltrato infantil, TEC y diagnóstico por imágenes
}

\author{
$M^{\mathrm{a}}$ ELIANA EBERHARD F. ${ }^{1,4}$, GEORGETTE POSE L. ${ }^{2,5}$, \\ XIMENA MORA D. ${ }^{4}, \mathrm{M}^{\mathrm{a}}$ FRANCISCA VERGARA A. ${ }^{3}$ \\ 1. Servicio de Anestesiología y Pabellón Hospital Roberto del Río. \\ 2. Servicio de Radiología, Hospital San Juan de Dios. \\ 3. Interna Medicina. Facultad de Medicina Clínica Alemana Santiago - Universidad del Desarrollo. \\ 4. Anestesiología y Unidad de Dolor, Clínica Alemana. \\ 5. Departamento de Imagenología, Clínica Alemana.
}

\begin{abstract}
\section{Child abuse, traumatic brain injury and image diagnosis}

Non-accidental Traumatic Brain Injury (TBI) in children is a significant social problem, which can leave significant permanent neurological sequelae, especially in children under 2 yo. This review shows the clinical symptomatological spectrum, which is sometimes quite subtle. It also provides the basis for use of images modalities for its study, to confirm a precise diagnosis.

(Key words: Child abuse, traumatic brain injury).

Rev Chil Pediatr 2011; 82 (3): 231-237

\section{RESUMEN}

El traumatismo encéfalo craneano de origen no accidental en niños, constituye un problema grave que afecta a nuestra sociedad y que puede dejar secuelas neurológicas permanentes especialmente en menores de dos años. Como una manera de situar el problema, hemos realizado una revisión con el objetivo de conocer el espectro de la sintomatología clínica del traumatismo encéfalo craneano, a veces muy sutil y fundamentar el rol de las distintas modalidades neuroradiológicas para precisar y confirmar su diagnóstico.

(Palabras clave: Maltrato infantil, Traumatismo encéfalo craneano).

Rev Chil Pediatr 2011; 82 (3): 231-237
\end{abstract}

Trabajo recibido el 26 de octubre de 2010, devuelto para corregir el 09 de diciembre de 2010, segunda versión el 01 de marzo de 2011, aceptado para publicación el 19 de marzo de 2011.

Correspondencia a:

Dra. M. Eliana Eberhard F.

E-mail: eberhard.mariaeliana@gmail.com 


\section{Introducción}

El maltrato infantil, entendido como toda forma de maltrato físico y/o emocional, abuso sexual, abandono o trato negligente, explotación comercial o de otro tipo, del que resulte un daño real o potencial para la salud, la supervivencia, el desarrollo o la dignidad del niño en el contexto de una relación de responsabilidad, confianza o poder, constituye uno de los problemas más graves y dramáticos que afecta a nuestra sociedad. Dentro de los maltratos físicos graves, están los traumatismos encéfalo craneanos (TEC) ${ }^{1-4}$.

Las lesiones cerebrales severas en menores de 2 años son frecuentemente secundarias a maltrato y son la causa más común de muerte. Su presentación clínica puede ser equivoca o pasar desapercibida, por eso es importante sospecharlo clínicamente, estudiarlo adecuadamente y conocer el espectro de hallazgos imagenológicos, algunas veces muy sutiles.

El maltrato infantil es un problema creciente del que sólo vemos la punta del iceberg, la magnitud del problema es mucho mayor. Hay pocos pero consistentes estudios en Chile que avalan este hecho, como el informe de la UNICEF, 1994. De 1533 niños que cursaban $8^{\circ}$ año básico, el $62 \%$ refirió haber sido víctima de violencia física, de las cuales 34,2\% fueron graves. De este grupo sólo el 10\% llegó al hospital y sólo el $1 \%$ denunció el hecho².

Estadísticas extranjeras (USA) demuestran que 1,5 millones de niños son maltratados al año. De éstos, 3000 mueren por lesiones cerebrales. El 10\% de las parálisis cerebrales son secundarias a maltrato y un $10 \%$ de éstas son objeto de maltrato ${ }^{3,4}$. Los traumatismos craneanos graves con lesión cerebral, constituyen la causa más frecuente de discapacidad.

En un estudio de 173 niños quienes sufrieron de un trauma no accidental y que estaban clínicamente sintomáticos, se demostró que en el 31\% de los casos hubo diagnóstico erróneo en primera instancia los que incluían: TEC de origen accidental, síndrome convulsivo, sepsis e infección viral ${ }^{5}$.

En una revisión de 100 niños menores de 2 años con trauma craneano los autores encontraron evidencias de trauma de origen no ac- cidental en un 24\% y sospechoso en un 32\% ${ }^{6}$.

Entre los años 1988 y 1997 se comparó casos de trauma de etiología accidental y no accidental en menores de 5 años. El porcentaje de admisiones por causa de origen no accidental fue de un 23,8\%. El promedio de edad en este grupo fue menor al grupo de etiología accidental (12.8 versus 27.5 meses). También se vio un mayor porcentaje de antecedentes de lesiones previas (27,8\% versus $0,07 \%)$. Los traumas de origen no accidental se producían más frecuentemente en la casa que aquellos de origen accidental (88,4 \% vs 54,9\%). Había un mayor porcentaje de injuria toráxica (12,5\% vs 4,5\%) y de lesiones abdominales $(11,4 \%$ vs $6,8 \%)$, las lesiones intracraneanas eran más frecuentes (68\% vs 23,5\%) al igual que las lesiones múltiples (47,6\% vs 39,7\%) y existía un mayor porcentaje de ingresados a las unidades de cuidados intensivos (42,5\% vs 26,9\%). También había una mayor proporción de muertes $(12,7 \% \text { vs } 2,6 \%)^{3-7}$.

En relación a la edad, otra revisión, concuerda con lo anterior, revelando que de 453 niños ingresados al servicio de urgencia con el diagnóstico de trauma craneano severo, entre los años 1996 y 1998, 125 eran menores de 2 años. Concluyen que los TEC graves son la primera causa de muerte y fuente de secuelas neurológicas severas. En este grupo etario cuando hubo sospecha de maltrato, se confirmó en el $45 \%$ de los casos. En los menores de 6 meses, la causa más frecuente de maltrato es el síndrome de niño sacudido y otras causas no precisadas $^{8}$.

La terminología ha cambiado con el mejor conocimiento de las causas biomecánicas de la injuria. En 1972, Caffey definió una forma específica de maltrato al que denominó "whiplash shaken-baby syndrome” o "síndrome del latigazo" para explicar la asociación de hemorragias intracraneanas (subdurales o subaracnoídeas), fracturas por tracción metafisiarias y hemorragia retiniana ${ }^{9,10}$.

En estudios biomecánicos, al usar un modelo de cabeza infantil mantenida por los hombros se demostró que la magnitud de la desaceleración angular (rotacional) es 50 veces mayor cuando el cráneo golpea fuertemente una superficie que aquel provocado por la sa- 
cudida como único evento y sólo se alcanza la injuria al momento del impacto. Cuando la superficie es blanda, la fuerza del impacto se disipa y puede no asociarse a signos visibles de trauma externo. Es la desaceleración rotacional súbita experimentada por el cerebro y los vasos y no la fuerza especifica de contacto lo que provoca la injuria intracraneal. Esta fuerza angular o rotacional, es distinta a las generadas en casi la totalidad de los casos de trauma accidental. El estudio clínico, radiológico y de anatomía patológica de la mayoría de los niños maltratados tienen evidencias de impacto ciego en la cabeza ${ }^{11,12}$. Es así como el término "Síndrome de sacudida- impacto" o "ShakingImpact Syndrome" reflejaría mejor el mecanismo responsable de las lesiones cerebrales, el cual puede resultar en el daño anóxico- isquémico debido a alteraciones metabólicas y circulatorias $^{13}$.

Las fuerzas que se producen durante los juegos de rutina o caídas de bajas alturas son insuficientes para causar este síndrome. Las fuerzas que realmente lo producen son resultado de una fuerza rotacional mayor, que claramente exceden a aquellas encontradas en las actividades corrientes.

Como una manera de situar el problema, hemos realizado una revisión con el objetivo de conocer el espectro de su sintomatología clínica del traumatismo encéfalo craneano, a veces muy sutil y fundamentar el rol de las distintas modalidades neuroradiológicas para precisar y confirmar su diagnóstico.

\section{Estudio diagnóstico}

El maltrato infantil se debe sospechar cuando existe historia clínica no concordante, retardo en la consulta o consulta reiterada por trauma, lesiones antiguas no tratadas o en sitios poco comunes ${ }^{14}$.

Su presentación clínica puede ser extremadamente engañosa y variable, como presencia de irritabilidad, vómitos, crisis de cianosis, convulsiones o encefalopatía recurrente. Los diagnósticos erróneos incluyen: TEC de origen accidental, síndrome convulsivo, sepsis y causa de origen viral ${ }^{5}$.
Cuando el examen neurológico esta alterado en un niño, debemos tener presente la posibilidad de maltrato, especialmente en menores de dos años. Debemos buscar alteración en el examen neurológico incluyendo el estudio de fondo de ojo para descartar la presencia de hemorragia retiniana que se asocia en un alto porcentaje con sacudidas violentas; alteración de la radiografía de cráneo y otras evidencias de trauma extracraneano ${ }^{15}$.

Las imágenes neuroradiológicas juegan un rol muy importante en el diagnóstico de maltrato. Estas incluyen la Radiografía de cráneo, Tomografía Computada (TC) y Resonancia Magnética (RM)

\section{Radiografía de cráneo}

Es de elección para detectar fractura craneanas y constituye un documento objetivo de trauma externo. Es importante saber que la ausencia de fractura no excluye la presencia de otras lesiones ${ }^{16}$.

En general la radiografía de cráneo por maltrato presenta una incongruencia entre el tipo de fractura y el mecanismo relatado. La historia clínica o presencia de alteraciones asociadas en el niño ayudan en el diagnóstico diferencial inicial.

La fractura lineal simple se ve en los traumas accidentales como en los provocados. Las que mayormente se han asociado a trauma de origen no accidental, son: fracturas múltiples (relacionadas a trauma reiterado), fracturas estrelladas (secundarias a impacto con objeto contundente), fractura conminuta (más de un fragmento), fractura occipital (ya que requiere de una enorme fuerza de impacto) y fracturas de cualquier tipo asociadas a signos de hipertensión endocraneana (separación de suturas y ensanchamiento de los surcos).

Es importante mencionar que niños con y sin fractura de cráneo presentan el mismo porcentaje de daño intracraneano, por lo tanto, será el examen neurológico el que determinará si se continuará el estudio imagenológico con TC o RM.

\section{Tomografía computada (TC)}

La tomografía (TC) es el método de elección para el estudio en sospecha de trauma 
craneano de origen no accidental. Alford recomienda TC cerebral en niños que presenten fractura craneana aún cuando haya ausencia de signos neurológicos, lo que es controvertido pues existen trabajos que no han demostrado un valor adicional en aquellos menores de 2 años con fractura de cráneo y examen neurológico normal ${ }^{17,18}$. El Colegio Americano de Radiología lo recomienda en todo niño menor de 2 años que sufra un trauma craneal ${ }^{19}$.

La TC es ampliamente disponible y muy sensible en la detección de sangre fresca y edema. Las lesiones intracraneanas que se pueden detectar son hemorragia, edema y encefalopatía hipóxico-isquémica.

\section{Hemorragias}

Las hemorragias pueden ser cerebrales o extracerebrales. Estas últimas generalmente son hematomas subdurales o hemorragias subaracnoídeas. Los hematomas epidurales son poco comunes en traumas no accidentales, pero los subdurales en niños pueden tener similar apariencia. Los hematomas subdurales presentan distintas densidades de acuerdo a su antigüedad. Los agudos aparecen hiperdensos (más blancos que la sustancia gris), los subagudos (entre 7 y 20 días) aparecen isodensos y aquellos crónicos (más de 20 días) aparecen hipodensos en relación al parénquima. Se recomienda el uso de contraste endovenoso para evaluar la cronicidad de un hematoma subdural, pues es posible identificar después del séptimo día la presencia de una membrana interna que delinea el hematoma ${ }^{20}$. Un hematoma subdural con componentes de baja y alta densidad puede representar un fenómeno crónico con re-hemorragia. Esto, asociado a edema cerebral y hemorragia subaracnoídea, es sugerente de hematoma subdural hiperagudo. La alta densidad representa el coágulo y la baja densidad la sangre no coagulada. Si estos hallazgos se presentan en un niño sin antecedentes de lesión accidental de alto impacto, se debieran considerar como un trauma provocado.

Los hematomas subdurales se localizan entre los hemisferios o infratentorialmente. Los interhemisféricos, como único hallazgo son altamente específicos de trauma provocado ${ }^{21}$. Los bilaterales en ausencia de fractura craneal y asociado a hemorragia retineana también son altamente sospechosos de abuso. Si existe más de un hematoma subdural y si se visualizan en distintas etapas de antigüedad, esto incrementa la sospecha de trauma craneal no accidental ${ }^{22}$.

Es muy importante revisar los exámenes previos al momento del ingreso. Los hematomas subdurales interhemisféricos pequeños pueden confundirse con un seno sagital prominente. La hoz cerebral normal puede aparecer brillante en comparación al resto de un cerebro edematoso. Las hemorragias intraventriculares por desgarro de las venas ependimarias son poco frecuentes. Las hemorragias cerebrales usualmente corresponden a lo que se denominan lesiones con disrupciones axonales difusas, las que se localizan en la interfase entre la sustancia gris y la sustancia blanca, tractos y comisuras de la sustancia blanca tales como el cuerpo calloso. Son de mal pronóstico y generalmente llevan a una disfunción cerebral.

\section{Edema}

El edema cerebral puede ser de difícil diagnóstico, debido a que la sustancia blanca inmadura en los niños dificulta su interpretación. Los signos tempranos son difíciles de ver y el seguimiento es fundamental para su comprobación. Los hallazgos típicos son la pérdida de la diferenciación entre la sustancia gris y blanca, con disminución de la atenuación de la corteza. En casos severos los ganglios basales, el cerebelo, tálamo y el tronco cerebral son relativamente respetados y pueden verse brillantes comparados con el resto. Esto ha sido denominado "el signo reverso" o "el signo del cerebelo blanco" que refleja la pérdida de la diferenciación entre la sustancia gris y blanca con conservación de la densidad del cerebelo y tronco encéfalo, el cual puede aparecer hiperdenso en comparación a la disminución de la densidad del resto del cerebro ${ }^{23,24}$. Normalmente esto refleja una causa no accidental de origen extra craneal, causada por hipoxia, tales como estrangulamiento o sofocación. Hay una alta asociación de este hallazgo con abuso cuando coexiste con hematoma subdural, pero no es específico y también puede verse en asfixia, paros cardíacos y en trauma accidental.

El edema cerebral puede ser reversible o 
evolucionar a la isquemia. Se acepta que los hallazgos son reversibles en la medida que no hay desviación de la línea media u oclusión de vasos.

\section{Encefalopatía hipoxico isquemica}

La encefalopatía hipóxico isquémica, puede provocar infartos con distribución de uno o múltiples territorios arteriales o todo un hemisferio. El mecanismo exacto de estos permanece inexplicado ${ }^{24}$. Las lesiones pueden ser confinadas a los ganglios basales o por el contrario, los respetan.

\section{Resonancia magnética (RM)}

La RM es especialmente sensible para demostrar presencia de pequeñas hemorragias subdurales, contusiones y laceraciones corticales pequeñas invisibles en la TC, siendo las que dejan más secuelas neurológicas ${ }^{1-16}$.

La RM tiene una resolución anatómica superior y ausencia de artefactos provocados por el cráneo especialmente en la fosa posterior. Se recomienda siempre cuando hay sospecha de trauma no accidental y la TC no demuestra anormalidades, pues esto no excluiría la presencia de hematomas subdurales.

Esta modalidad permite determinar con exactitud la edad de los hematomas subdurales y diferenciar los crónicos de higromas quísticos formados por líquido cefalorraquídeo muy similares en la TC. La secuencia T2 de gradiente tiene la habilidad de evaluar regiones de susceptibilidad magnética diferentes tales como la hemosiderina, por lo que es útil en la detección de hemorragias antiguas por disrupción axonal, invisibles en la TC.

Las imágenes de difusión son útiles en la detección de daño axonal difuso en la etapa precoz. Es de elección para evaluar el daño cerebral después de una injuria aguda. La necrosis cortical puede ser el resultado de sofocación y/o estrangulación y puede ser detectada en las imágenes por resonancia en la secuencia T1.

La presencia de hemorragias con nivel líquido hace sospechar la presencia de un trauma repetido lo, que es frecuente en un niño maltratado.

\section{Diagnóstico diferencial radiológico}

El error radiológico más frecuente es una TC negativa. Un diagnóstico diferencial particularmente difícil es el agrandamiento benigno del espacio subaracnoídeo o espacio subaracnoídeo complaciente ${ }^{25}$. La razón exacta de este hallazgo aun es desconocida, aunque clínicamente existe un incremento en la circunferencia del cráneo, cuyo seguimiento es favorable con normalización dentro de los 18 meses. El hecho puntual es que niños con espacio subaracnoídeos amplios tienen un riesgo mayor de hemorragias subdurales o subaracnoídeas, que no necesariamente corresponden a un trauma no accidental, siendo difícil determinar su verdadera causa.

La ausencia de lesiones asociadas y de colecciones sanguíneas están a favor de un agrandamiento benigno del espacio subaracnoídeo. Es importante pensar la posibilidad y excluir hemorragias debido a desórdenes por coagulopatía intravascular diseminada, infecciones hemorrágicas y sangramientos en niños sometidos a terapia anticoagulante. En un estudio reciente fueron revisados 26 niños con lesiones cerebrales accidentales (sepsis, síndrome de muerte súbita, paro cardíaco, inhalación de humo y convulsiones). Las hemorragias intracraneales fueron encontradas en 5 de ellos, pero nunca un hematoma subdural ${ }^{25}$.

Las alteraciones de la coagulación al manifestarse como hemorragias constituyen un diagnóstico diferencial.

Las infecciones, tales como sepsis y meningitis, podrían asociarse con infartos multifocales, edema cerebral y ocasionalmente hemorragia.

Los traumas craneales accidentales en menores de 2 años raramente se asocian a lesiones intracraneanas y caídas de menos de un metro, sólo ocasionalmente producen lesiones severas $^{26}$.

\section{Evaluación oftalmológica}

La detección de la hemorragia retiniana es vital en la investigación de un niño con sospecha de trauma de origen no accidental. El estudio oftalmológico debiera realizarlo una persona capacitada ${ }^{27}$. La mayoría de las hemo- 
rragias traumáticas post natales son de origen no accidental, sin embargo, también se han descrito después de traumas craneales accidentales severos y también se pueden ver en otras condiciones generales, tales como, incremento de la presión venosa, sepsis, coagulopatía y ciertos desordenes metabólicos ${ }^{28,29}$. Las resucitaciones cardiopulmonares y las convulsiones, si se tratan en forma adecuada, raramente causan hemorragia retiniana.

El diagnóstico diferencial es extenso y existen diferencias cualitativas y cuantitativas. En un trauma craneal no accidental la hemorragia es usualmente bilateral y puede involucrar todas las capas de la retina ${ }^{30,31}$. La hemorragia está localizada típicamente en el polo posterior, pero puede extenderse hacia la periferia. También pueden observarse en traumas severos, hemorragia del vítreo, retinosquisis traumática, pliegues retineales y desprendimientos retineales. En niños bajo un año la mayoría de las hemorragias retineales son frecuentemente descritas en traumas no accidentales, siendo menos severas en niños mayores ${ }^{32}$.

El mecanismo sacudida-impacto es el que mejor explica la hemorragia retineal y su severidad está estrechamente relacionada con la severidad de la injuria intracraneal ${ }^{33}$.

\section{Conclusiones}

Un número importante de los traumas craneanos graves en preescolares, son de origen no accidental. Debe sospecharse en todo niño, particularmente aquel menor de 2 años con historia poco clara, asociado o no a alteración del examen neurológico. Deberá realizarse un cuidadoso examen físico incluido fondo de ojo, radiografía de cráneo y del resto del esqueleto así como TC y RM cerebral. Normalmente una revisión esquelética debiera realizarse y repetirse después de los 10 a 14 días, para demostrar lesiones óseas en fase de consolidación.

La TC y la RM de cerebro pueden mostrar no solamente lesiones típicas, sino patognomónicas de maltrato. El hematoma subdural, la hemorragia subaracnoídea y la hemorragia retiniana aislada, son marcadamente más comunes en lesiones por maltrato infantil.

La determinación de diferentes edades de un hematoma puede reflejar un trauma repetido característico de abuso, lo cual pudiera ser un hallazgo muy importante desde el punto de vista médico legal. Es importante saber que la TC puede ser negativa por lo que si clínicamente existe sospecha de una lesión neurológica debiera indicarse una RM.

En los niños maltratados el mecanismo de lesión cerebral es variable. Las fracturas o heridas en el cuero cabelludo, han recibido impacto directo, sin embargo, aquellos sin evidencias de lesiones externas pueden presentar daño intracraneano, provocadas por mecanismos de sacudida o latigazo.

Como médicos enfrentamos una gran responsabilidad en su diagnóstico e implicancias legales. Debemos desplegar todo nuestro esfuerzo y trabajo para sensibilizar tanto al personal de salud como al resto de la población de manera de enfrentar este problema, tomando conciencia de su significado y coordinar nuestros esfuerzos con instituciones que permitan encontrar un camino claro para tomar las decisiones correctas y oportunas en beneficio del niño y de su grupo familiar.

\section{Agradecimientos}

Nuestros agradecimientos a los Drs. Patricio Miller y Cesar García por sus valiosos aportes en cada una de sus especialidades, las que ayudaron a una mayor claridad y precisión del manuscrito.

\section{Referencias}

1.- Pose G: Lesiones cerebrales en el maltrato infantil. Revista Chilena de Radiología 1997; 3 (2): 74-83.

2.- Larraín HS, Bascuñan DC: Maltrato infantil y relaciones familiares en Chile Análisis comparativo 19942000-2006. Rev Chil Pediatr 2008; 79 Supl (1): 64-79.

3.- DiScala C, Sege R, Li G, Reece RM: Child abuse: A 10-year retrospective. Arch Pediatr Adolesc Med 2000; 154: 16-22.

4.- Carney NA, Chesnut R, Kochanek PM: Guidelines for the acute medical management of severe traumatic brain injury in infants, children and adolescents. A supplement to: Pediatric Critical Care Medecine 2003; 4 (3). 
5.- Jenny C, Hymel KP, Ritzen A, Reinert SE, Hay TC: Analysis of missed cases of abusive head trauma. J Am Med Assoc 1999; 281: 621-6.

6.- Caffey J: The Whiplash shaken infant syndrome: manual shaking by the extremities with whiplash-induced intracranial and intraocular bleedings, linked with residual permanent brain damage and mental retardation. Pediatrics 1974; 54: 396-403.

7.- Zenel J, Goldstein B: Child abuse in the padiatric intensive care unit. Crit Care Med 2002; 30 (1): S515-23.

8.- Giroux JD, Sizun J, Cabelguen C, Guillois B, Alix D, De Parscau $L$ : Traumatismes crâniens graves du nourrisson: nécessité d’une prise encharge hémodynamique pré-hospitalière. Réanim Soins Intensifs Med Urg 1993; 9: 35-40.

9.- Duhaime AC, Christian WC, Rorke LB, Zimmerman $R A$ : Nonaccidental head injury in infants - the "ShakenBaby Syndrome”. N England J Med 1998; 338 (25): 1822-29.

10.- Chiesa A, Duhaime AC: Abusive Head Trauma. Pediatr Clin North Am 2009; 56 (2): 317-31.

11.- Duhaime AC, Gennarelli TA, Thibault LE, Bruce DA, Margulies SS, Wiser R: The shaken baby syndrome: a clinical, pathological, and biomechanical study. J Neurosurg 1987; 66: 409-15.

12.- Alexander R, Sato $Y$, Smith W, Bennett T: Incidence of impact trauma with cranial injuries ascribed to shaking. Am J Dis Child 1990; 144: 724-6.

13.- Duhaime AC, Alario AJ, Lewander WJ, et al: Head Injury in very young children: mechanisms, injury types and ophthalmologic findings in 100 hospitalized patients younger than 2 years of age. Pediatrics 1992; 90: 17985.

14.- Guías de Maltrato Infantil-2003: Hospital de Niños Dr. Roberto del Río Moya Hernán, Olivari Francisco, Ulloa Fresia.

15.- Wilkins B: Head Injury-abuse or accident? Archives if Disease in Childhood 1997; 76 (5): 393-6.

16.- Merten DF, Osborne DR, Radkowski MA, Leonidas JC: Craniocerebral trauma in the child abuse syndrome: radiological observation. Pediatr Radiol 1984; 14: 272-7.

17.- Sato Y, Yuh WTC, Smith WL, Alexander RC, Kao SCS, Ellerbroek CJ: Head Injury in Child Abuse: Evaluation with MR Imaging. Radiology 1989; 173: 653-7.

18.- Mogbo KI, Slovis TL, Canady AI, Allasio DJ, Arfken C: Appropriate imaging in children with skull fractures and suspicion of abuse. Radiology 1998; 208: 521.

19.- Demaerel P, Casteels I, Wilms G: Cranial imaging in child abuse. Eur Radiol 2002; 12: 849-57.

20.- Harwood-Nash DC: Abuse to the pediatric central nervous system. Am J Neuroradiol 1992; 13: 569-75.

21.- Barnes PD, Robson CD: CT findings in hyperacute nonaccidental brain injury. Pediatr Radiol 2000; 30: 74-81.

22.- Trenchs V, Curcoy AI, Navarro R, Pou J: Subdural Haematoma and Physical Abuse in the First Two Years of Life. Pediatr Neurosurg 2007; 43: 352-7.

23.- Rao P, Carty H, Pieree A: The acute reversal sign: comparison to medical and non-accidental injury patients. Clin Radiol 1999; 54: 495-501.

24.- Han BK, Towbin RB, Courtens-Myers G, McLaurin RL, Ball WS Jr: Reversal sing on CT: effect of anoxic/ ischemic cerebral injury in children. Am J Roentgenol 1990, 154: 361.

25.- Wilms G, Vanderschueren G, Demaerel PH, et al: CT and MR in infants with pericerebral collections and macrocephaly: benign enlargement of the subarachnoid spaces versus subdural collections. AJNR Am J Neuroradiol 1993; 14 (4): 855-60.

26.- Chadwick DL, Chin S, Salerno C: Death from falls in children. How far is fatal? J Trauma 1991 31: 1353-5.

27.- Maguire S, Pickerd N, Farewell D, Mann M, Tempest V, Kemp AM: Which clinical features distinguish inflicted from non-inflicted A systematic review. Arch Dis Child 2009; 94 (11): 860-7.

28.- Gilliland MG, Luckenbach MW, Chenier TC: Systemic and ocular findings in 169 prospectively studied child deaths: retinal haemorrhages in non-accidental injury. Forensic Sci 1994; 68: 117-32.

29.- Johnson DL, Braun D, Friendly D: Accidental Head Trauma and Retinal Hemorrhage. Neyrosurgery 1993; 33 (2): 231-5.

30.- Tyagi AK, Willshaw HE, Ainsworth JR: Unilateral retinal hemorrhages in non-accidental injury. Lancet 1997; 349: 1224.

31.- Drack AV, Petronio J, Capone A: Unilateral retinal hemorrhages in documented cases of child abuse. Am J Ophthalmol 1999; 128: 340-4.

32.- The Ophthalmology Child Abuse Working Party: Child abuse and the eye. Eye 1999; 13: 3-10.

33.- Glick JC, Staley K: Inflicted traumatic brain injury: advances in evaluation and collaborative diagnosis. Pediatr Neurosurg 2007; 43 (5): 436-41. 